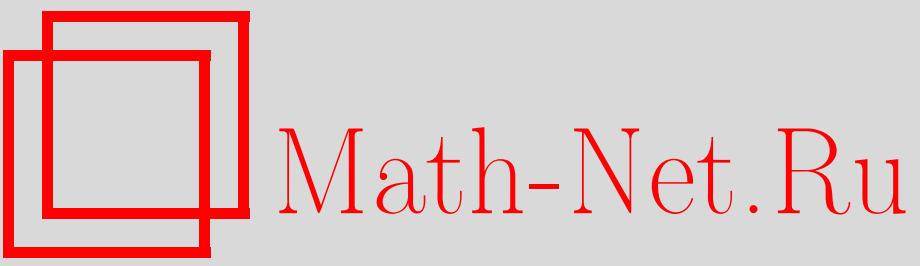

А. А. Котельникова, О лакунах в спектре одного эллиптического оператора на периодической сетке, УМH, 2004, том 59, выпуск 5, 153-154

DOI: https://doi.org/10.4213/rm780

Использование Общероссийского математического портала Math-Net.Ru подразумевает, что вы прочитали и согласны с пользовательским соглашением

http://www . mathnet.ru/rus/agreement

Параметры загрузки:

IP : 54.80 .73 .141

26 апреля 2023 г., 17:59:27 


\section{О ЛАКУНАХ В СПЕКТРЕ ОДНОГО ЭЛЛИПТИЧЕСКОГО ОПЕРАТОРА НА ПЕРИОДИЧЕСКОЙ СЕТКЕ}

\section{А. А. КотЕЛЬниковА}

На плоскости рассматривается периодический связный графф $F=F_{1} \cup F_{0}$ с ячейкой периодичности $\square=[0,1)^{2}$ (см. рис. 1). На каждом ребре граффа $F$ зададим периодическую нормированную меру $\mu$, пропорциональную мере Лебега. Определим также $\varepsilon$-периодическую меру $\mu_{\varepsilon}$ равенством $\mu_{\varepsilon}(B)=\varepsilon^{2} \mu\left(\varepsilon^{-1} B\right)$ для любого борелевского множества $B \subset \mathbb{R}^{2}$ и, следуя [1] и [2], введем соболевское пространство $H^{1}\left(\mathbb{R}^{2}, d \mu_{\varepsilon}\right)$.

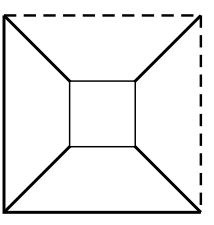

$F \cap \square$

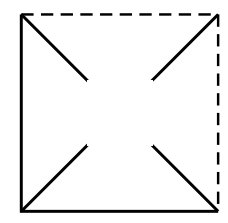

$F_{1} \cap \square$

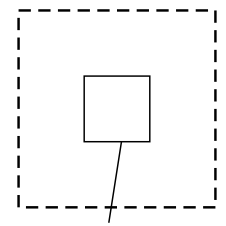

$F_{0} \cap$

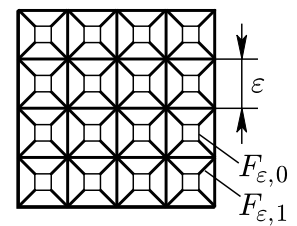

$F_{\varepsilon}$

Рис. 1

В связи с изучением моделей "двойной пористости" [1] ставится задача исследования поведения при $\varepsilon \rightarrow 0$ решений $u_{\varepsilon} \in H^{1}\left(\mathbb{R}^{2}, d \mu_{\varepsilon}\right)$ уравнения

$$
-\operatorname{div}\left(a_{\varepsilon} \nabla u_{\varepsilon}\right)+u_{\varepsilon}=f \in C_{0}^{\infty}\left(\mathbb{R}^{2}\right), \quad a_{\varepsilon}(x)= \begin{cases}1, & x \in F_{\varepsilon, 1}, \\ \varepsilon^{2}, & x \in F_{\varepsilon, 0},\end{cases}
$$

где $F_{\varepsilon, 1}, F_{\varepsilon, 0}$ - гомотетические сжатия множеств $F_{1}, F_{0} ; F_{\varepsilon}=F_{\varepsilon, 1} \cup F_{\varepsilon, 0}$.

Мы рассматриваем также более реалистическую задачу, когда ребра граффа $F_{\varepsilon}^{h}=F_{\varepsilon, 1}^{h} \cup F_{\varepsilon, 0}^{h}$ имеют толщину $h=h(\varepsilon) \rightarrow 0$ при $\varepsilon \rightarrow 0$. При этом аналогом (1) служит

$$
\mathscr{A}_{\varepsilon}^{h} u_{\varepsilon}^{h}+u_{\varepsilon}^{h}=f, \quad \mathscr{A}_{\varepsilon}^{h}=-\operatorname{div}\left(a_{\varepsilon}^{h} \nabla\right),
$$

где $a_{\varepsilon}^{h}=1$ на $F_{\varepsilon, 1}^{h}$ и $a_{\varepsilon}^{h}=\varepsilon^{2}$ на $F_{\varepsilon, 0}^{h}$. Через $\mu_{\varepsilon}^{h}$ обозначим соответствующую рассматриваемой задаче $\varepsilon$-периодическую меру. Решения $u_{\varepsilon}^{h} \in H^{1}\left(\mathbb{R}^{2}, d \mu_{\varepsilon}^{h}\right)$ имеют сильньй двухмасштабньй предел:

$$
\lim _{\varepsilon \rightarrow 0} \int_{\mathbb{R}^{2}}\left|u_{\varepsilon}^{h}(x)-u\left(x, \varepsilon^{-1} x\right)\right|^{2} d \mu_{\varepsilon}^{h}=0,
$$

где $u(x, y) \in L^{2}\left(\mathbb{R}^{2} \times \square, d x \times d \mu\right)$ периодична по $y$ и принадлежит классу

$$
V \equiv\left\{u(x, y)=u_{1}(x)+u_{0}(x, y) ; u_{1} \in H^{1}\left(\mathbb{R}^{2}\right), u_{0} \in L^{2}\left(\mathbb{R}^{2}, H_{\mathrm{per}}^{1}(\square, d \mu)\right),\left.u_{0}\right|_{F_{1}}=0\right\} .
$$

ОпредЕлЕниЕ. Функция $u=u_{1}+u_{0} \in V$ называется решением предельного уравнения, если для любой функции $\varphi=\varphi_{1}+\varphi_{0} \in V$ справедливо интегральное тождество

$$
\frac{1}{2} \int_{\mathbb{R}^{2}} \nabla u_{1} \cdot \nabla \varphi_{1} d x+\int_{\mathbb{R}^{2}} \int_{\square} \nabla y u_{0} \cdot \nabla_{y} \varphi_{0} d x d \mu+\int_{\mathbb{R}^{2}} \int_{\square} u \varphi d x d \mu=\int_{\mathbb{R}^{2}} \int_{\square} f \varphi d x d \mu .
$$

Здесь $\nabla y$ - градиент функции, заданной на графе $F_{0}$.

Левая часть (3) задает замкнутую симметрическую билинейную форму на $V$, и потому предельное уравнение записьвается в виде $\mathscr{A} u+u=f$, где $\mathscr{A}$-самосопряженный оператор в гиль-

Работа выполнена при финансовой поддержке Российского фонда фундаментальных исследований (грант № 02-01-00114). 
бертовом пространстве $H=\bar{V}$ - замькании $V$ в $L^{2}\left(\mathbb{R}^{2} \times \square, d x \times d \mu\right)$. Предельное уравнение сводится к системе

$$
\left\{\begin{array}{l}
\mathscr{A}_{1} u_{1}+u_{1}+\int_{F_{0} \cap \square} u_{0} d \mu=f \text { в } \mathbb{R}^{2}, \\
\mathscr{A}_{0} u_{0}+u_{1}+u_{0}=f \text { на } F_{0} \cap \square .
\end{array}\right.
$$

Здесь $-2 \mathscr{A}_{1}=\Delta_{x}$ - классический лапласиан в $\mathbb{R}^{2}, \mathscr{A}_{0}$ - оператор в $L^{2}\left(F_{0}, d \mu\right)$, равньй прямой сумме лапласианов, отвечающих задачам Дирихле на каждом ребре $F_{0}$. Спектр оператора $\mathscr{A}_{0}$ состоит из собственных значений $\alpha_{k}=(2 k)^{2} \pi^{2} / l^{2}, k=0,1,2, \ldots, \omega_{k}=(2 k-1)^{2} \pi^{2} / l^{2}$, $k=1,2, \ldots$, где $l \in(0,1)$ - длина ребра $F_{0}$. Положим

$$
\beta(s)=s+s^{2} \sum_{n=1}^{\infty} \frac{c_{n}^{2}}{\omega_{n}-s}, \quad c_{n}=\int_{F_{0} \cap \square} \varphi_{n} d \mu, \mathscr{A}_{0} \varphi_{n}=\omega_{n} \varphi_{n}, \quad \int_{F_{0} \cap \square} \varphi_{n}^{2} d \mu=1
$$

и обозначим нули функции $\beta(s)$ через $\nu_{k}(k \in \mathbb{N})$.

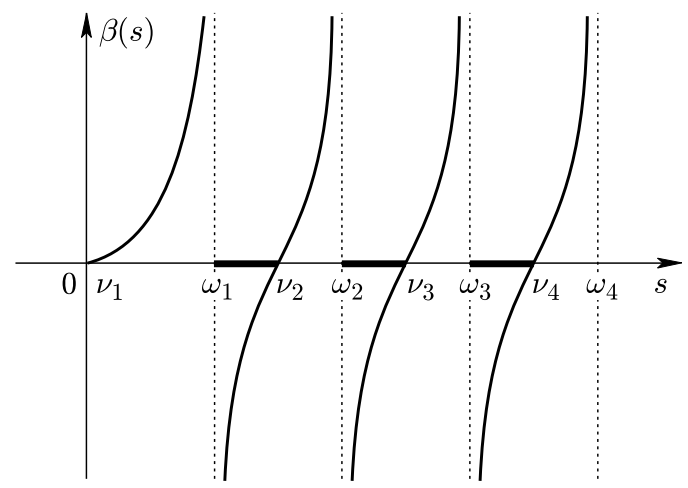

Рис. 2

Tеорема 1. Непрерывный спектр предельного оператора $\mathscr{A}$ состоит из обгединения отрезков $\bigcup_{k=1}^{\infty}\left[\nu_{k}, \omega_{k}\right]$. Если $0<l \leqslant \frac{1}{3}$, то дискретный спектр оператора $\mathscr{A}$ пуст. Если $\frac{3}{4}<l<1$, то при достаточно большом $k$ собственное значение $\alpha_{k}$ попадает $в$ интервал $\left(\omega_{k}, \nu_{k+1}\right)$ и, таким образом, принадлежит дискретному спектру $\mathscr{A}$.

На рис. 2 выделена бесконечная система интервалов, где функция $\beta(s)$ отрицательна. Эти интервалы могут содержать лишь точки дискретного спектра оператора $\mathscr{A}$ из множества $\left\{\alpha_{k}\right\}$, в которых $\beta\left(\alpha_{k}\right)<0$. Смежные отрезки, на которых $\beta(s) \geqslant 0$, принадлежат непрерьвному спектpy $\mathscr{A}$.

TEOpema 2. Пусть $h(\varepsilon) \rightarrow 0$ nрu $\varepsilon \rightarrow 0$. Тогда спектр оператора $\mathscr{A}_{\varepsilon}^{h}$ сходится no Xaусдорфу к спектру оператора $\mathscr{A}$. В частности, спектр оператора $\mathscr{A}_{\varepsilon}^{h}$ имеет лакуньи, число которых неограниченно растет при $\varepsilon \rightarrow 0$.

Резултаты, аналогичные теоремам 1 и 2 , для случая ограниченной области и $h=0$ установлены в [3]. Автор благодарит В.В. Жикова за постановку задачи и обсуждение полученных результатов.

\section{СПИСОК ЛИТЕРАТУРЫ}

[1] В. В. ЖКиков // Матем. сб. 2000. Т. 191.№ 7. С. 31-72. [2] В. В. ЖКиков // Труды семинара им. И. Г. Петровского. Вып. 22. М.: Изд-во МГУ, 2002. С. 105-120. [3] А. A. Kotelnikova, V. V. Zhikov. Spectral aspects in double-porosity models // Abstracts of the International Workshop ANASTHEM, Saint Petersburg, Russia, 2000. P. 38-41. 